\title{
Remote Laser Laboratory: First Demonstration
}

\author{
Worldwide Substitution for Laser Engineering Lab \\ http://dx.doi.org/10.3991/ijoe.v8iS1.1891 \\ Igor Titov $^{1,2}$, Olga Smirnova ${ }^{1}$, Alexander Glotov ${ }^{1}$ and Alexander Golovin ${ }^{1}$ \\ ${ }^{1}$ R\&D Institute of Radioelectronics and Laser Engineering, BMSTU, Moscow, Russia \\ ${ }^{2}$ EPFL Lausanne, Switzerland
}

\begin{abstract}
Remote Laser Laboratory (RLL) is hosted at Bauman Moscow State Technical University (BMSTU), Russia. Local laser engineering lab can be classified as complex, expensive and dangerous facility. Potentially it poses risks both for students and for laboratory itself when operated by unskilled users. This paper presents arguably the most optimal solution fitting great in both e-learning and safe remote operation paradigms: Remote Laser Laboratory [1]. The emphasis here is placed on software part but hardware solutions are also described.
\end{abstract}

Index Terms-computer aided instruction; expensive equipment; e-learning; hazardous environment; laser remote laboratory; student experiments; labicom.

\section{INTRODUCTION}

Recently our laser engineering laboratory has gone through extensive upgrade of safety regulations standards that made its operation as educational lab almost impossible (at least for large groups of students). This happened due to revision of related legislation and regulations for all University laser labs. At the same time curriculum change for courses participating in

laser lab did not occur. This led to desperate need of finding a new way to conduct the same lab sessions. Perfect solution here is remote lab: on one hand students fulfill the same experiments on actual physical equipment; on the other hand they do not interact with such hazardous environments as laser radiation and high voltage equipment at all. Such solution also increases throughput of educational laboratory allowing having cleaner optical environment at the same time. All these benefits are com-

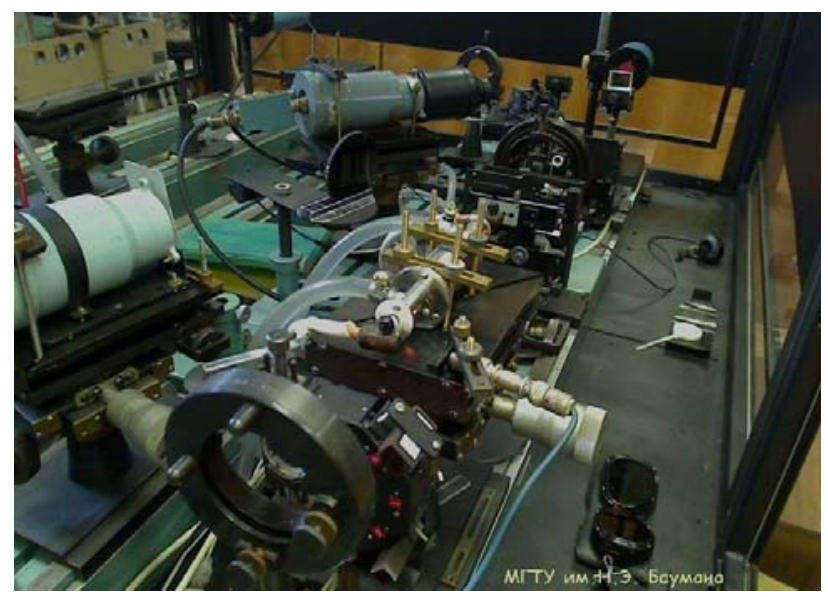

Figure 1. Web-camera view of RLL physical equipment on optical laser bench bined with those of general benefits of generic remotelaboratory, i.e. flexible timetable, worldwide accessibility etc. Thus, laser engineering laboratory at BMSTU was transformed into remote lab and beta version of RLL is presented in this paper. Operation of RLL will be demonstrated on exp.at'11 conference both from perspective of a student and of the RLL-server. Live experiments during the exhibition at the conference will be carried out.

\section{RLL SOFTWARE}

RLL software implements client-server architecture: lab server communicates with client application through TCP sockets. Lab server controls physical equipment and manages TCP connections whereas client is a rich internet application dealing with displaying data in the user's browser.

\section{A. Client-side software}

RLL client application is hosted by web-server [2] and is available 24/7. RLL client is a Flash application (swf file), that means that it can be viewed with any modern browser on any modern platform. The only prerequisite is Adobe Flash Player installed in user's browser.

\section{B. Server-side software}

Sever-side RLL is written in NI LabView. It is a heart of RLL although invisible by most users: lab server executes control algorithms, security interlocks, communication with client-side application, manages all TCPconnections, handles graphical user interface for lab administrator and provides control over different laboratory settings. Graphical user interface of RLL server side con-

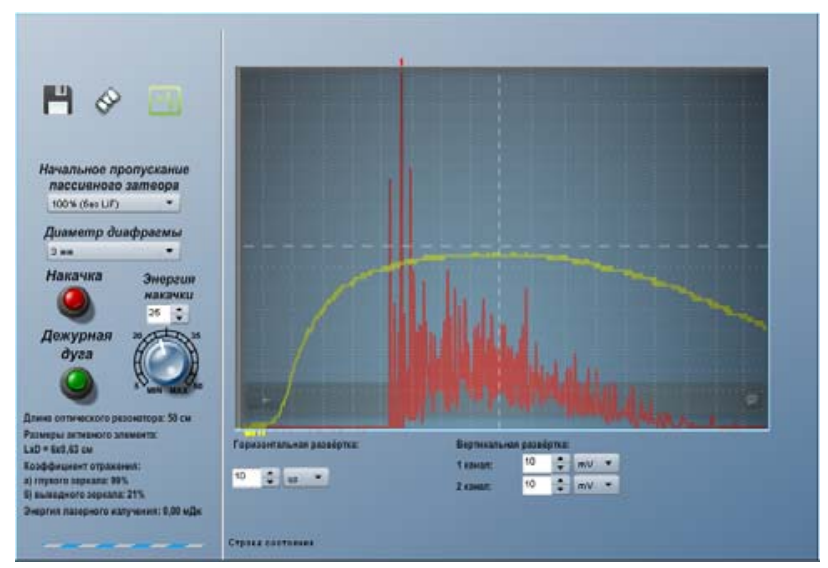

Figure 2. RLL client graphical user interface 


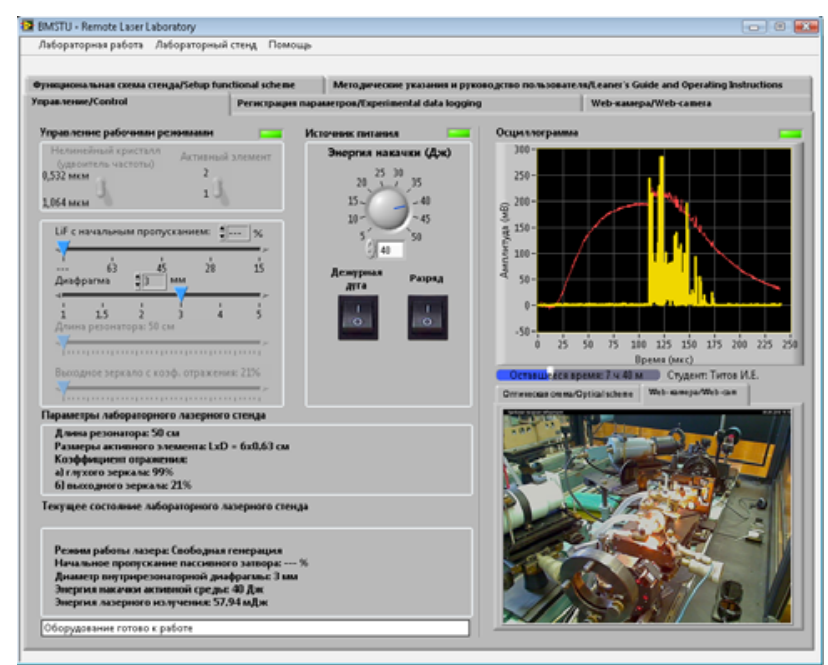

Figure 3. RLL lab-server main tab

sists of tabs. Each of them specializes on some particularaspect of RLL operation: advanced control capabilities, web-cameras’ settings, RLL general settings, current optical scheme on laser bench, etc. For example, one can see oscilloscope and web-camera images on the main tab with equipment control but there are advanced settings for web-camera and for oscilloscope on corresponding tabs.

RLL server takes an advantage of LabView multithread capabilities distributing processes between different parallel loops. RLL control algorithm takes into account current equipment in use preventing potentially dangerous simultaneous operation of conflicting equipment. For instance without such measures one might have an attempt to generate laser pulse while aperture diaphragm is moving, thus damaging it. RLL server software includes also alarm system (activates in case of a hardware malfunction), logging system (stores all actions of all users during lab session), interface for laboratory administrator.

\section{Information security}

On the client side information security is provided by using 'sandbox' which is safe environment in which Flash application operates. On the server side there are three layers of security: hardware level (system of interlocks), access layer (only registered users can control real equipment), logging layer (all users' actions are monitored, analyzed and stored). Between RLL lab server and RLL client there is a layer of transmitted data protection: TCP packets are encrypted with AES 256 (Advanced Encryption Standard 256-bit key) symmetric key algorithm.

\section{RLL HARDWARE AND EQUIPMENT}

\section{A. Quantron}

Quantron represents gain medium (YAG:ND) and cooling cavity in one mechanical structure. The system uses forced water cooling while power supply unit is turned on.

\section{B. Web-cameras}

RLL can use up to five commercial USB web-cameras with a resolution of 800x600 pixels. View from the quantron-side camera is presented on figure 1.

\section{Control Unit and Power Supply Unit}

RLL control unit interacts with optical functional elements (Q-switch, diaphragm, non-linear crystal, etc.) and communicates with a computer through USB interface. Power supply unit controls the pumping energy, discharge frequency, pilot arch and initiates discharges event (and therefore, laser shot).

\section{Oscilloscope}

RLL uses USB digital $200 \mathrm{MHz}$ oscilloscope with 1 $\mathrm{GS} / \mathrm{s}$ sampling rate allowing to register signals up to $2 \mathrm{~ns}$.

\section{E. Joulemeter}

Energy measurement unit (joulemeter) incorporates photoelectric cathode, capacitance integrator, ADC and microcontroller. Measured energy is transmitted to the RLL lab server.

\section{F. Step-motors and actuators}

Step-motors and actuators control functional parts of RLL instrumentation, i.e. Q-switches, optical filters, diaphragm diameter, mirrors and non-linear crystal. Each of these elements is a custom-made electromechanical structure.

\section{CONCLUSIONS}

RLL replaces local engineering laboratory at BMSTU [3] giving flexibility and safety of remote operation. RLL will have been used and tested in quantum electronics and laser engineering courses since 2012 academic year.

\section{REFERENCES}

[1] I. Titov, O. Smirnova, A. Glotov and A. Golovin, "Remote Laser Laboratory: lifebuoy for laser engineering curriculum”, International Journal of Online Engineering, in press

[2] http://www.labicom.net/labs/rll

[3] http://www.bmstu.ru

\section{AUTHORS}

Igor Titov, Olga Smirnova, Alexander Glotov and Alexander Golovin aer with R\&D Institute of Radioelectronics and Laser Engineering, BMSTU, Moscow, Russia.

Igor Titov is also with EPFL Lausanne, Switzerland.

This work is a description of a demonstration given during $1^{\text {st }}$ Experiment@ International Conference, 17/18 November 2011 in Lisbon, Portugal. Manuscript received 05 December 2011. Published as resubmitted by the authors 20 January 2012. 